\title{
A one-stop perineal clinic: our eleven-year experience
}

\section{Osanna Yee Ki Wan ${ }^{1,2} \cdot$ Annika Taithongchai $^{1} \cdot$ Susana I. Veiga ${ }^{1} \cdot$ Abdul H. Sultan ${ }^{1,3} \cdot$ Ranee Thakar $^{1,3}$ (D)}

Received: 6 April 2020 / Accepted: 17 June 2020 / Published online: 2 July 2020

(C) The Author(s) 2020

\begin{abstract}
Introduction and hypothesis The perineal clinic is a dedicated setting offering assessment for various childbirth-related presentations including obstetric anal sphincter injuries (OASIs), perineal wound complications, pelvic floor dysfunction and other conditions such as female genital mutilation(FGM). We describe the clinical presentation and outcomes of women from a tertiary perineal clinic based on data collected over an 11-year period.

Methods This is a retrospective observational study. A one-stop outpatient service was offered to all women who sustained OASIs (postnatally and antenatally in a subsequent pregnancy), perineal complications (within 16 weeks postpartum), FGM and/ or peripartum symptoms of urinary/anal incontinence or prolapse. Assessment included history with validated questionnaires, examination and anal manometry and endoanal ultrasound when appropriate. Outcomes were compared among different grades of OASIs. Management of each type of presentation was reported with outcomes.

Results There were 3254 first attendance episodes between 2006 and 2016. The majority (58.1\%) were for OASIs, followed by perineal wound complications. Compared to the lower grades, the higher grades of OASI were associated with poorer outcomes in terms of symptoms, investigations and complications. Women with OASIs had unrelated symptoms such as urinary incontinence, perineal pain and wound infections that needed further intervention. A high proportion(42\%) of wound complications required further specialist management.

Conclusion We describe a dedicated, one-stop perineal clinic model for antenatal and postnatal women for management of perineal and pelvic floor disorders. This comprehensive and novel data will enable clinicians to better counsel women regarding of outcomes after OASI and focus training to minimize risks of morbidities.
\end{abstract}

Keywords Anal incontinence $\cdot$ Female genital mutilation $\cdot$ Obstetric anal sphincter injuries $\cdot$ Perineal clinic $\cdot$ Perineal trauma Perineal wound complications

\section{Introduction}

Perineal trauma is the most common complication during vaginal delivery, occurring in $42 \%$ of women [1]. Obstetric anal sphincter injuries (OASIs) are the most severe form with potentially devastating effects on a mother's quality of life $[2,3]$. Up to $50 \%$ of women suffer from perineal pain and dyspareunia following OASIs, and these symptoms can last

Ranee Thakar

ranee.thakar@nhs.net

1 Department of Obstetrics and Gynaecology, Croydon University Hospital, 530 London Road London CR7 7YE UK

2 Department of Obstetrics and Gynaecology, Prince of Wales Hospital, The Chinese University of Hong Kong, Hong Kong Hong Kong

3 St George's University of London, Cranmer Terrace London UK for years $[4,5]$. There is also mounting evidence that the management and outcome of wound complications, persistent perineal pain or postpartum dyspareunia in those without OASIs remain unknown, although serious problems greatly affecting the physical and mental health of women have been reported [6].

These women frequently do not receive the dedicated care they need because of lack of knowledge in both healthcare professionals and women as well as poor care. The Royal College of Obstetricians and Gynaecologists (RCOG) have recommended that all women sustaining OASIs should have an assessment around 6 to 12 weeks postpartum by clinicians with a special interest in OASI where possible [7]. However, a survey of clinical leads of hospitals in the UK demonstrated that only $32 \%$ offered a dedicated outpatient clinic follow-up for women after OASIs [8]. Furthermore, postpartum women with perineal complications not related to OASIs should also be expected to have an opportunity for a similar assessment. 
Women with female genital mutilation (FGM) have been reported to have poorer obstetric outcomes in both lowresource and resource-rich countries [9], with a contributing factor being lack of knowledge and experience of healthcare professionals. However, when appropriate antenatal counselling and management are available via a dedicated perineal clinic, obstetric outcomes showed no difference from those without FGM [10]. Peripartum women may also suffer from urinary incontinence, anal incontinence (AI) and prolapse symptoms affecting their quality of life, which may often be neglected because of lack of help available or the assumption that these symptoms are a "normal" consequence of childbirth, without appropriate investigation or assessment.

Various different models of perineal clinics have been reported. These include clinics led by consultant urogynaecologists [11-13], consultant obstetrician and gynaecologists $[13,14]$, with some triaged or assisted by specialized midwives/nurses [11,12,14, 15], and clinics led by a specialist midwife [16]. Formats of clinics include: one-stop clinics offering all necessary investigations and referrals [17, 18], a standard outpatient set-up where investigations are performed either at a later date [13-15] or prior to initial assessment [12], those where only limited investigations are available [15] or simple telephone follow-up [16]. The maximum number of patients reported in any series is up to 400 [14] and the longest duration of experience of perineal clinics reported was 4 years [11]. The targeted groups of patients seen also varied from those only focusing on postnatal OASIs [15] to including patients with perineal pain $[11,14]$ or AI outside the peripartum period of all ages [14], while some included AI only in antenatal or postnatal women $[11,13,15]$. Different management protocols have also been used for the management of OASI patients [19-21].

We described the clinical presentation and outcomes of women seen in our tertiary one-stop perineal clinic based on data collected over an 11-year period.

\section{Materials and methods}

This is a retrospective observational study over an 11-year period between January 2006 to December 2016. A dedicated perineal clinic was established at Croydon University Hospital, London, UK, in 2002. All patient data were entered into a database prospectively. This weekly clinic provided a one-stop service for women, run by a consultant urogynaecologist with a concomitant perineal wound clinic, staffed by a trained specialist perineal midwife. Appropriate investigations as well as management of symptoms and counselling for future mode of delivery (MOD) when necessary were all offered at the same attendance [19]. Women are referred from within our institution, from local general practitioners or from surrounding hospitals. Referrals are accepted for women in both the postpartum period following OASIs and antepartum period with history of OASIs in a subsequent pregnancy, postpartum perineal wound complications such as infection, dehiscence, pain or dyspareunia, women with FGM either pregnant or outside of pregnancy, new symptoms of AI in the antenatal or postnatal period, complaints suggestive of a missed clinical diagnosis of OASIs or other symptoms of pelvic floor dysfunction in the peripartum period such as urinary incontinence, prolapse or sexual dysfunction. Other types of referrals include suspected genital tract fistula or other previous perianal/perineal surgery in pregnant women where MOD may be affected. The postpartum women were seen up to 16 weeks postnatally, after which they would be instead referred to the urogynaecology clinic or combined Pelvic Floor Clinic. Information regarding the clinic was sent to the women with their appointment letter.

A detailed history was obtained including demographic data (age, parity, ethnicity), MOD, obstetric details, degree of perineal tear and presence of vaginal, urinary or bowel symptoms. Perineal and anal sphincter trauma was classified using the Sultan classification [2, 7]. Severity of AI was assessed using the validated modified St. Mark's incontinence score (SMIS), which ranged from 0 (no symptoms) to 24 (severe incontinence) [22]. Urinary incontinence was assessed using the validated International Consultation on Incontinence modular Questionnaire for Urinary Incontinence-Short Form (ICIQ-UI_SF), ranging from 0 (no symptoms) to 21 (severe incontinence) [17, 23]. Women underwent vaginal examination to assess wound integrity, size of the perineal body $(\mathrm{cm})$ and pelvic floor muscle contraction using the Modified Oxford Scale [24]. Appropriate investigations such as anal manometry (AM) and endoanal ultrasound scan (EAUS) were performed in all women with history of OASIs irrespective of symptoms or grade of OASI as previously reported $[18,19]$ and when clinically indicated. Defects in the sphincter muscle were defined as $\geq 30^{\circ}$ disruption (equivalent to $>1 \mathrm{~h}$ on the clock face) in the external anal sphincter (EAS) at the deep, superficial and/or subcutaneous levels or the internal anal sphincter (IAS) as previously described [22].

\section{Patient management}

Perineal pain and/or dyspareunia, urinary incontinence and pelvic organ prolapse were managed as per structured local evidence-based or best practice protocols (available on www. perineum.net). Women with a history of OASIs were counselled regarding subsequent MOD, based on presence of symptoms and investigation findings as previously reported [19], as well as ensuring they were practising pelvic floor exercises (PFE). Perineal wound infection was diagnosed on clinical examination as evidenced by local 
perineal tenderness, erythaema, exudate, odour and oedema, with or without pyrexia. Wound dehiscence was diagnosed when there was gaping of the perineal wound $(>0.5 \mathrm{~cm})$. Wound swabs were taken for culture and sensitivities and oral antibiotics commenced (local protocol as agreed with microbiology being amoxicillin/clavulanic acid), loose sutures removed, perineal washout performed if necessary and perineal hygiene advice given). In cases of systemic infection, intravenous antibiotics were recommended with twice daily perineal washout. Women with wound dehiscence were counselled regarding the options of wound re-suturing or healing by secondary intention given that there is a lack of evidence of best practice for management of wound dehiscence [25]. Wound infections were initially reviewed weekly every 2-3 weeks and for wound dehiscence review would be every 2 weeks once the infection is controlled.

Women who had undergone FGM were questioned regarding associated urinary, bowel, sexual and infective symptoms. Pregnant women requiring de-infibulation were advised to have it done in the second trimester. Other FGM complications such as cysts and de-infibulation in non-pregnant women would be managed accordingly.

Women with perineal pain or dyspareunia were assessed for abscesses or fistulae by vaginal and/or rectal examination and EAUS or perineal ultrasound. Management includes examination under anaesthesia, antibiotics, incision and drainage of the abscess or removal of irritant undissolved suture materials as necessary. Persisting granulation tissue was treated with silver nitrate application.

In cases of persistent perineal or scar tenderness, perineal massage with topical 5\% lidocaine ointment was advised. If there was associated dyspareunia, the women were asked to apply it about $30 \mathrm{~min}$ prior to coitus. If the problem still persisted, a cocktail of $10 \mathrm{ml} 0.5 \%$ bupivacaine, $1500 \mathrm{IU}$ hyaluronidase and $40 \mathrm{mg}$ Depo-Medrone (methylprednisolone acetate) solution was injected to the site of maximal tenderness. A second or third injection could be repeated after a minimum of 6 weeks. Vaginal dilators, psychosexual counselling, myofascial release and/ or perineoplasty were recommended when indicated. Women with an anal fissure were given advice with regard to relieving constipation by having a high fibre diet along with fibre supplements such as Fybogel (Ispaghula Husk) and oral Lactulose in combination with topical anal application of lidocaine ointment prior to defecation and perineal hygiene advice. Local application of glycerine trinitrate $0.4 \%$ ointment was also prescribed to relax IAS spasm, frequently associated with anal fissures.

\section{Ethics approval}

All investigations were part of routine clinical practice, and therefore ethical approval was not required.

\section{Statistical analysis}

The data were analysed using IBM SPSS statistics version 22 . Descriptive analysis was used to study the demographics, symptoms of AI, urinary incontinence and their respective SMIS and ICIQ-UI scores, and the incidence of wound complications in women with OASIs. The Fisher's exact test, chisquared test, Student's t-test and Mann-Whitney U test were used for statistical comparisons. $p<0.05$ was taken as statistical significance.

\section{Results}

There were 3254 first attendances between January 2006 and December 2016. The mean age was $30.3 \pm 5.5$ years, and the mean body mass index was $25.1 \pm 4.7 \mathrm{~kg} / \mathrm{m}^{2}$. The median parity was 1 [interquartile range (IQR) 1,2 ]. The median follow-up was 10 weeks (IQR 5, 17) after delivery.

The women were of mixed ethnicity, including $40.2 \%$ White, 20.6\% Black African, 10.6\% Asian Indian, 15.0\% other Asian and $13.6 \%$ other ethnicity. The majority (70\%) were patients from our institution, $20 \%$ were referred by the general practitioner, and $10 \%$ were tertiary referrals from other hospitals. Most (58.9\%) were seen during the postpartum period, $35.6 \%$ were seen during the antenatal period while the remaining $5.5 \%$ were unrelated to pregnancy.

The primary reasons for referral to the perineal clinic are listed in Table 1.

\section{OASI}

Urinary incontinence was the most common symptom, with up to $16.5 \%$ having a mean ICIQ-UI score of $1.9 \pm 3.8$, followed by flatal incontinence in $11.8 \%$, with a mean SMIS of $1.9 \pm 3.7$ (Table 2). Overall incidence of perineal pain and dyspareunia in women with OASI was $6.7 \%$ and $2.4 \%$, respectively. Treatment options included application of silver nitrate for granulation tissue and perineal massage (15.6\%) for perineal pain with two requiring a steroid cocktail injection for persisting perineal pain. Wound infection and dehiscence were found in $2.7 \%$ and $3.3 \%$ in women with OASI, respectively. Ten women $(1.0 \%)$ had re-suturing of their perineal wound for dehiscence and $11(1.0 \%)$ women underwent a secondary sphincter repair (one $3 \mathrm{a}$, two $3 \mathrm{~b}$, five $3 \mathrm{c}$ tears, one unspecified third-degree and two 4th-degree tears). Urogenital prolapse was found in $1.0 \%$, with five apical and anterior compartment prolapses, two anterior and posterior compartment prolapses, two posterior compartment prolapses only and one apical compartment prolapse only. There were eight women with fistula: one perineorectal, two perineovaginal, three rectovaginal ( 1 with a concomitant perineovaginal 
Table 1 Primary reasons for referral to perineal clinic

Reason for referral

Number of women (\%) $(N=3254)$

Obstetric anal sphincter injury (OASI)

$1892(58.1)$

OASI managed at 3 months postnatally

Third-degree tear

Third-degree tear $3 a$

Third-degree tear $3 b$

Third-degree tear $3 \mathrm{c}$

Unclassified third-degree tear

Fourth-degree tear

Rectal buttonhole tear

OASI managed antenatally in a subsequent pregnancy $\quad 742$

Perineal complications

Wound infection

Wound dehiscence

Perineal pain/dyspareunia

Other perineal wound problems such as haematoma/swelling/migrating stitches/labial adhesions 66

Perineal masses such as varicosities, Bartholin's cyst

Extensive perineal tear (not OASIS) requiring debriefing

Female genital mutilation (FGM)

FGM type 1

FGM type 2

FGM type 3

FGM type 4

Patient declined examination/unclassified FGM

No definite FGM when examined

Peripartum pelvic floor problem

Urinary incontinence

Urogenital prolapse

Voiding dysfunction

Bowel problem (without a definite history of OASI)

Anal incontinence

Faecal incontinence

Flatal incontinence

Faecal urgency

Others: constipation, per-rectal bleeding

Referred for suspected missed or undiagnosed OASIS after delivery

Due to deficient perineum in postpartum examination by GP

Delivery with uncertain degree of perineal trauma or patient worried about third-degree tear

Referred for advice on mode of delivery

Perianal disease/ulcerative colitis/fistula

History of anal surgery, e.g., sphincterotomy, fissurectomy

History of fistula repair

History of perineal refashioning

Vaginal septum/vaginal hymen ring

$\mathrm{FGM}=$ female genital mutilation, $\mathrm{GP}=$ general practitioner, $\mathrm{OASI}=$ obstetric anal sphincter injury

fistula and 1 after a rectal button-hole tear) and another two trans-sphincteric, all requiring surgical intervention.
As demonstrated in Tables 2 and 3, compared to women who sustained a third-degree tear, women with a fourth- 


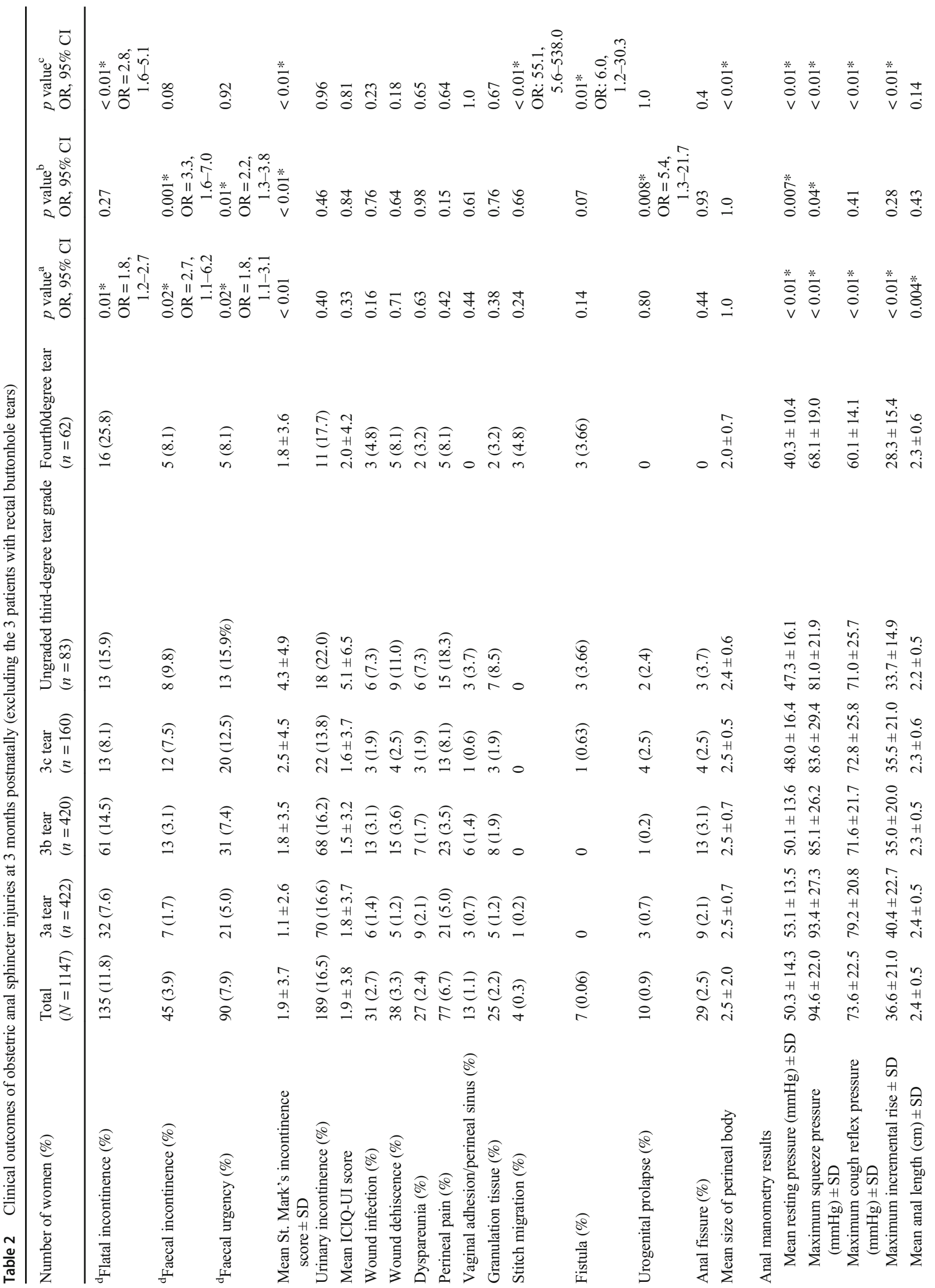




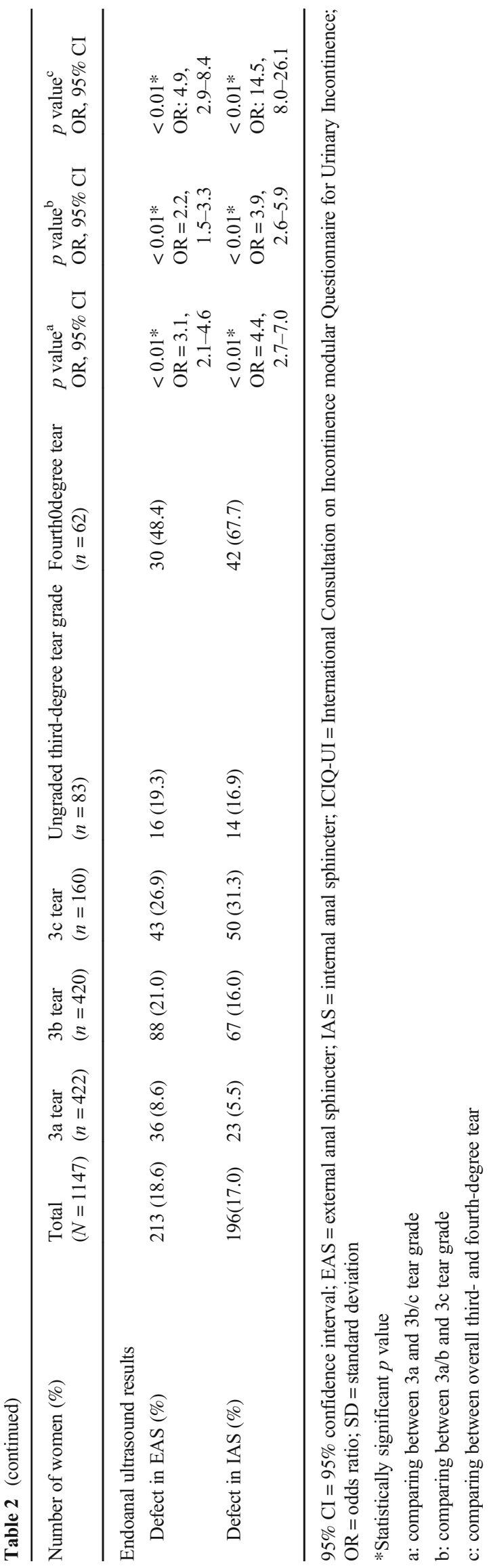

degree tear had almost three times higher risk of having flatal incontinence ( $p<0.01)$, higher mean SMIS $(3.9 \pm 5.7$ vs. 1.8 $\pm 3.6, p<0.01)$, more complications such as migrated sutures [OR (odds ratio) $=55,95 \%$ CI (confidence interval) $5.6-$ 538.0] and more referrals for further management to the joint pelvic floor clinic for colorectal input. Furthermore, women with clinically diagnosed IAS injury, i.e., $3 \mathrm{c}$ tear, were 2.2 times more likely to have faecal urgency, 3.3 times more faecal incontinence, 5.4 times more likely to have urogenital prolapse, had lower AM pressures and were more likely to have persistent EAS (2.0 times) and IAS (4.0 times) defects. Moreover, when comparing individual compartment prolapse, there no statistical differences were found between different degrees of tear. OASI did not have a statistically significant association with urogenital prolapse after adjustment by foetal size and mode of delivery in multivariate analysis. No differences were found among different grades of third-degree tears for other wound outcomes (Tables 2 and 3).

There were 742 women seen antenatally in a subsequent pregnancy. Around $21.6 \%$ had symptoms of AI with the mean SMIS being $1.9 \pm 4.6$. Persistent EAS and IAS defects were found in $21.8 \%$ and $21.0 \%$, respectively. Of these women, $11.3 \%$ were advised for caesarean section, and $78.0 \%$ were advised that there was no contraindication to vaginal delivery, with a low threshold for episiotomy. The remaining were either undecided during the consultation or the decision was unknown because it was dependent on other obstetric factors such as foetal size or placental location. The obstetric outcomes for women following their subsequent pregnancies have been reported previously [19].

\section{Postnatal perineal wound complications}

Perineal complications were the second most common reason for attendance to the perineal clinic, namely wound infection (30.9\%), perineal pain (29.5\%), wound dehiscence (27.4\%), other perineal masses, stitch migration or haematoma formation $(12.3 \%)$. More than $42 \%$ of them required further intervention, including 64 women (8.4\%) who underwent secondary re-suturing of the perineal wound, $119(15.6 \%)$ silver nitrate application, $12(1.6 \%)$ suture removal, $41(5.4 \%)$ scar refashioning, 58 (7.6\%) and 17 (2.2\%) local anaesthetic and steroid cocktail injections for pain relief, respectively, and 13 $(1.7 \%)$ vaginal dilators.

\section{Female genital mutilation}

The most common type of FGM presentation was type 2 (partial or total excision of the clitoris and labia minora with or without removal of the labia majora): 242 (76.1\%) were seen antenatally, 62 (19.5\%) postnatally, and 14 outside of the peripartum period. In total, 17 women underwent surgical treatment, including 7 de-infibulations performed antenatally, 4 
de-infubulations intrapartum, 1 postnatal perineal reconstruction and a further 5 de-infibulations in non-pregnant women.

\section{New bowel symptoms without OASIs}

Most of these women (134) had symptoms of AI; one had constipation and the other per rectal bleeding. The majority $(80.2 \%)$ were referred postnatally. Their mean SMIS was $10.0 \pm 6.0$. All women presenting with symptoms of AI and no history of OASI were offered AM and EAUS for assessment. EAS and IAS defects were found in 38 (36.8\%) and 27 (19.5\%) women, respectively; 40 (29.4\%) women were diagnosed to have had clinically missed OASIs at the time of delivery and were managed as previously described [18]. Functional anal length, mean maximum resting pressure, mean maximum squeeze pressure and mean maximum cough reflex pressure were $1.7 \pm 1.1 \mathrm{~cm}, 38.0 \pm 24.4 \mathrm{mmHg}, 77.0 \pm$ $26.5 \mathrm{mmHg}$ and $69.9 \pm 23.1 \mathrm{mmHg}$, respectively. All were taught PFE, 25 (18.8\%) were referred for biofeedback for bowel symptoms, $6(4.5 \%)$ required loperamide for symptom control, $6(4.5 \%)$ were offered secondary repair as they had completed their family and $1(0.8 \%)$ had sacral nerve modulation.

\section{Peripartum urinary incontinence}

The mean ICIQ-UI score was $11.6 \pm 4.9$ for those presenting with a primary complaint of UI. Up to $95.3 \%$ were advised to practise PFE, 20.9\% underwent bladder retraining, $7.0 \%$ had biofeedback, and $4.7 \%$ were prescribed anti-cholinergics. About one third had further follow-up with the urogynaecology nurse specialist and the remainder did not require further referral.

\section{Peripartum pelvic organ prolapse}

A clinical diagnosis of pelvic organ prolapse was confirmed in $22(73.3 \%)$ with $52.9 \%$ suffering from stage II and the remaining stage I pelvic organ prolapse by the Pelvic Organ Prolapse Quantifications System. Four women chose pessary insertion and two out of the four eventually opted for a surgical repair: one sacrohysteropexy and one anterior and posterior pelvic floor repair.

\section{Discussion}

We describe the clinical presentation and outcome of 3254 women seen in a dedicated one-stop perineal clinic based on data collected over an 11-year period. As far as we know, this is the largest reported series of patients in the literature attending the first dedicated one-stop perineal clinic with EAUS and AM. The most common reason for referral was OASIs, with the second being perineal wound complications, with a high proportion requiring further management. Women who sustained $3 \mathrm{~b}$ or more severe OASIs had significantly higher risks of symptoms of $\mathrm{AI}$, poorer performance on AM, persistent sphincter defects on EAUS and more complications such as fistula formation or need for a secondary sphincter repair.

Although many published studies evaluate AI after OASIs $[2,4,5,26]$, other associated complications, outcomes and management have not been described $[22,27]$. The large

Table 3 Treatment or procedures required for complications in women with obstetric anal sphincter injuries (excluding the 3 women with a rectal buttonhole tear)

\begin{tabular}{|c|c|c|c|c|c|c|c|c|c|}
\hline $\begin{array}{l}\text { Number of women } \\
(\%)\end{array}$ & $\begin{array}{l}\text { Total } \\
(\mathrm{N}=1147)\end{array}$ & $\begin{array}{l}3 \text { a tear } \\
(n=422)\end{array}$ & $\begin{array}{l}3 \mathrm{~b} \text { tear } \\
(\mathrm{n}=420)\end{array}$ & $\begin{array}{l}3 \mathrm{c} \text { tear } \\
(\mathrm{n}=160)\end{array}$ & $\begin{array}{l}\text { Ungraded 3rd- } \\
\text { degree tear }(n=83)\end{array}$ & $\begin{array}{l}\text { Fourth-degree } \\
\text { tear }(n=62)\end{array}$ & $\begin{array}{l}p \text {-value }^{\mathrm{a}} \\
\text { OR, 95\% CI }\end{array}$ & $\begin{array}{l}p \text {-value }{ }^{\mathrm{b}} \\
\text { OR, 95\% CI }\end{array}$ & $\begin{array}{l}p \text {-value }{ }^{\mathrm{c}} \\
\text { OR, } 95 \% \mathrm{CI}\end{array}$ \\
\hline${ }^{\mathrm{d}}$ Wound re-suturing & $10(0.9)$ & $2(0.5)$ & $5(1.2)$ & $1(0.6)$ & $1(1.2)$ & $1(1.6)$ & 0.33 & 0.79 & 0.42 \\
\hline $\begin{array}{l}{ }^{\mathrm{d}} \text { Secondary sphincter } \\
\text { repair }\end{array}$ & $11(1.0)$ & $1(0.2)$ & $2(0.5)$ & $5(3.1)$ & $1(1.2)$ & $2(3.2)$ & 0.09 & $\begin{array}{l}<0.01^{*} \\
\mathrm{OR}=9.0 \\
2.1-38.2\end{array}$ & 0.11 \\
\hline $\begin{array}{l}{ }^{\mathrm{d}} \text { Lidocaine gel for } \\
\text { perineal pain }\end{array}$ & $23(2.0)$ & $10(2.4)$ & $5(1.2)$ & $4(2.5)$ & $4(4.9 \%)$ & 0 & 0.34 & 0.54 & 0.63 \\
\hline${ }^{\mathrm{d}}$ Cocktail injection & 2 & 0 & 1 & 0 & 1 & 0 & 0.58 & 0.84 & 0.90 \\
\hline $\begin{array}{l}{ }^{\mathrm{d}} \text { Silver nitrate for } \\
\text { granulation tissue }\end{array}$ & $21(1.8)$ & $3(0.7)$ & $9(2.1)$ & $2(1.3)$ & $6(7.3 \%)$ & $1(1.6)$ & 0.12 & 0.86 & 1.0 \\
\hline${ }^{\mathrm{d}}$ Vaginal dilators & $5(0.4)$ & $1(0.2)$ & $2(0.5)$ & 0 & $1(1.2)$ & $1(1.6)$ & 0.76 & 0.45 & 0.24 \\
\hline${ }^{\mathrm{d}}$ Scar re-fashioning & $5(0.4)$ & $2(0.5)$ & $3(0.7)$ & 0 & 0 & 0 & 0.93 & 0.33 & 1.0 \\
\hline
\end{tabular}

$95 \% \mathrm{CI}=95 \%$ confidence interval $; \mathrm{OR}=$ odds ratio

*Statistically significant $p$-value

a: comparing between $3 \mathrm{a}$ and $3 \mathrm{~b} / \mathrm{c}$ tear grade

b: comparing between $3 \mathrm{a} / \mathrm{b}$ and $3 \mathrm{c}$ tear grade

c: comparing between overall third- and fourth-degree tear

d: chi-square test and Fisher exact tests are used 
number in our cohort provided us a unique opportunity to report this. We found that the degrees or grades of OASIs did not differ in the incidence of wound infection, dehiscence or pain. However, a significant proportion of women who sustained OASIs required further postnatal treatment for management of pain, granulation tissue, wound infection or dehiscence. This demonstrates the importance of offering these women the opportunity to be examined postnatally and enquiring about symptoms besides the specific assessment of bowel symptoms and sphincter integrity.

A significantly shorter mean size of the perineal body was found in women who sustained fourth-degree tears compared to those with third-degree tears $(2.0 \pm 0.7$ vs. $2.5 \pm 1.3, p<$ 0.01 ). This supports the importance of examining the perineal body during postpartum care, especially in cases of possible missed tears [18]. The reconstruction of the perineal body muscles is particularly important for supporting the sphincter repair as a short and deficient perineum can pose higher risks of OASI recurrence in future deliveries [3].

We found women with a $3 \mathrm{c}$ tear to be more likely to develop a fistula $(\mathrm{OR}=6.0,95 \% \mathrm{CI}=1.2-30.3)$ and undergo a secondary anal sphincter repair $(\mathrm{OR}=9.0,2.1-38.2)$, highlighting the importance of training in making the correct diagnosis of the full extent of injury as well as paying particular attention to adequate primary IAS repair [2, 22]. In addition, they are 5.4 times more likely to develop symptoms of urogenital prolapse, highlighting the importance of encouraging women to perform PFE.

In keeping with a previous study [17], we found that urinary incontinence was the most common concomitant symptom with OASIs. We acknowledge that this is a very select group of women with OASIs without a comparative control group and this could be a common transitory postnatal change. However, it does highlight the importance of advocating PFE to these women. In women without OASIs who presented with urinary incontinence, although having high ICIQ-UI scores, few required ongoing management after PFE indicating that implementing simple conservative treatments is often all that is necessary.

Reported rates of wound infection and dehiscence in women with perineal trauma vary between $0.1 \%-23.6 \%$ and $0.21 \%-24.6 \%$, respectively [6]. Specific data on wound complications in OASIs are limited [28]. We found the rate after OASI to be on the lower side $(2.7 \%$ infection and $3.3 \%$ dehiscence, respectively). This could be due to technique but also women with OASIs are prescribed antibiotics prophylactically at the time of repair [7]. Our local protocol advocates oral antibiotics for 3 days in addition to intra-operative intravenous antibiotics.

The second most common reason for attendance was perineal wound complications with a very high proportion requiring specific treatment for their symptoms and management options, which may not necessarily be available in other settings. Without a dedicated clinic, it is possible that the vast majority would have persistent symptoms without accessing help, but just suffering in silence. There are no internationally recognized guidelines on management of symptoms such as perineal pain, dyspareunia or wound dehiscence but we have developed protocols that have provided excellent standardized treatment (www.perineum.net). We advocated the use of short-term prophylactic antibiotics after repair of complex perineal tears but not simple tears. A recently published large multicentre randomized trial found that one prophylactic dose of antibiotic in women undergoing operative vaginal birth significantly reduced the rate of perineal infection [29].

The establishment of an open-access dedicated one-stop clinic enabled provision of evidence-based standardized care by experienced professionals. These clinic data show the multiple benefits to women of this type of service: the opportunity to explore the circumstances under which their perineal injury occurred, understand the extent of trauma and in many cases provide simple reassurance. A duty of candour and giving consistent advice to women is important from a medicolegal perspective particularly as this is an area of obstetrics where there is increasing litigation. It is also an arena where taboo topics such as FGM could be openly discussed and managed by experienced professionals with little to no barriers. This is particularly important for antenatal patients as it is a crucial time to identify these women and arrange appropriate treatment options.

The one-stop model avoids multiple visits to the hospital, an important consideration in postpartum women. We found that combined AM and EAUS performed by the same physician at one visit optimizes the management plan by correlating with symptoms enabling a holistic approach to women with clinically recognized and unrecognized OASIs [18]. With improved clinical detection of OASIs in recent years due to improved training there is also a risk of overdiagnosis, reported in one large series to be $7 \%$ [30].

We acknowledge the limitations of our article. We were unable to report data on women who have presented to other hospitals with their complications. While we were unable to report the incidence of wound complications in women with OASIs, women with other perineal trauma were not followed up routinely in our setup; therefore, establishing the total percentage of wound complications for all women was not possible. We also do not have a control group for comparison of outcomes and a set follow-up period to report on the natural history of these conditions. Some conditions might selfresolve over time, regardless of the intervention. In addition, ours is a tertiary referral centre and therefore may provide an over-representation of symptoms. We did not have patient satisfaction data over this period. In recent times, there has been an increased awareness of postpartum complications among healthcare professionals and patients. Hence, we could have witnessed higher rates of referrals. We appreciate that 
our findings and opinions are based on the experience of a single centre and our study population was heterogeneous, possibly making our results generalizable. However, the data were collected prospectively, limiting recall bias. Other strengths include the use of validated questionnaires, objective validated investigative tools and the use of standardized management protocols.

\section{Conclusion}

There is undoubtedly considerable attention directed toward antenatal women with regard to foetal and maternal conditions. By comparison, there is a relative disparity in postpartum care, particularly related to the pelvic floor and perineum. This article demonstrates the utility, justification and benefits of a dedicated, one-stop, perineal clinic for postpartum women who have specific problems related to their perineum, anal sphincters, vagina or pelvic floor. Apart from women who have sustained OASIs there are postpartum women who suffer bowel, bladder, prolapse and sexual problems and many of these women suffer in silence. In a system of universal healthcare and equality, one could argue that if a local institute is unable to provide the above model of care, then these patients should ideally be referred to a hospital able to provide the necessary investigations and management. These comprehensive and novel data enable clinicians to better counsel women regarding outcomes after OASI and other perineal problems, focus training to minimize risks of morbidities, justify the establishment of one-stop perineal clinics and enhance data collection for audit, research and development.

Author contributions OYK Wan: Data management and data analysis, manuscript writing.

A Taithongchai: Manuscript writing/editing.

SI Veiga: Data collection, Manuscript editing.

AH Sultan: Protocol/project development, Manuscript editing.

R Thakar: Protocol/project development, Manuscript editing.

\section{Compliance with ethical standards}

Financial disclaimers/conflict of interest Ranee Thakar is currently the President of the International Urogynecology Association. All other authors have no conflicts of interest to disclose.

Open Access This article is licensed under a Creative Commons Attribution 4.0 International License, which permits use, sharing, adaptation, distribution and reproduction in any medium or format, as long as you give appropriate credit to the original author(s) and the source, provide a link to the Creative Commons licence, and indicate if changes were made. The images or other third party material in this article are included in the article's Creative Commons licence, unless indicated otherwise in a credit line to the material. If material is not included in the article's Creative Commons licence and your intended use is not permitted by statutory regulation or exceeds the permitted use, you will need to obtain permission directly from the copyright holder. To view a copy of this licence, visit http://creativecommons.org/licenses/by/4.0/.

\section{References}

1. NHS Maternity Statistics, England 2017-18. NHS Digital. https:// digital.nhs.uk/data-and-information/publications/statistical/nhsmaternity-statistics/2017-18. Assessed 26 Mar 2020.

2. Sultan AH, Thakar R, Fenner DE. Perineal and anal sphincter trauma. London: Springer; 2007.

3. Kapoor DS, Thakar R, Sultan AH. Obstetric anal sphincter injuries: review of anatomical factors and modifiable second stage interventions. Int Urogynecology J. 2015;26:1725-34.

4. Fernando RJ, Sultan AH, Kettle C, Thakar R (2013) Methods of repair for obstetric anal sphincter injury. Cochrane Database Syst Rev. CD002866. https://doi.org/10.1002/14651858.CD002866. pub3.

5. Keighley MRB, Perston Y, Bradshaw E, Hayes J, Keighley DM, Webb S. The social, psychological, emotional morbidity and adjustment techniques for women with anal incontinence following obstetric anal sphincter injury: use of a word picture to identify a hidden syndrome. BMC Pregnancy Childbirth. 2016;16:275.

6. Jones K, Webb S, Manresa M, Hodgetts-Morton V, Morris RK. The incidence of wound infection and dehiscence following childbirth-related perineal trauma: a systematic review of the evidence. Eur J Obstet Gynecol Reprod Biol. 2019;240:1-8.

7. Royal College of Obstetricians and Gynaecologists (2015) The management of third- and fourth-degree perineal tears. Green-top guidelin no. 29. https://www.rcog.org.uk/globalassets/documents/ guidelines/gtg-29.pdf. Assessed 26 Mar 2020.

8. Ismail SIMF. The management of obstetric anal sphincter injuries (OASIS): a national postal questionnaire survey in hospitals in the UK. J Obstet Gynaecol. 2015;35:229-34.

9. Wuest S, Raio L, Wyssmueller D, Mueller MD, Stadlmayr W, Surbek DV, et al. Effects of female genital mutilation on birth outcomes in Switzerland. BJOG. 2009;116:1204-9. https://doi. org/10.1111/j.1471-0528.2009.02215.x.

10. Balachandran AA, Duvalla S, Sultan AH, Thakar R. Are obstetric outcomes affected by female genital mutilation? Int Urogynecology J. 2018;29:339-44.

11. Brincat C, Crosby E, McLeod A, Fenner DE. Experiences during the first four years of a postpartum perineal clinic in the USA. Int J Gynecol Obstet. 2015;128:68-71.

12. Fowler G, Williams A, Murphy G, Taylor K, Wood C, Adams E. How to set up a perineal clinic. Obstet Gynaecol. 2009;11:129-32.

13. Pretlove SJ, Thompson PJ, Toozs-Hobson PM, Radley S. The first 18 months of a new perineal trauma clinic. J Obstet Gynaecol. 2004;24:399-402.

14. Fitzpatrick M, Cassidy M, O'Connell PR, O'Herlihy C. Experience with an obstetric perineal clinic. Eur J Obstet Gynecol Reprod Biol. 2002;100:199-203.

15. Corry E, O'Connor E, Eogan M, Fitzpatrick M, O'Sullivan S, Imcha M. A dedicated Perineal clinic - an audit in support. Ir Med J. 2019;112:913.

16. Shawer S, Innayat S, Hutchinson H, Ballard P, Khunda A. Introducing a new service of a midwife-led telephone follow-up clinic for 3 a \& 3 b obstetric anal sphincter injury: retrospective service evaluation and patients' satisfaction survey. Pelviperineology. 2019;1:49-52.

17. Scheer I, Andrews V, Thakar R, Sultan AH. Urinary incontinence after obstetric anal sphincter injuries (OASIS) - is there a relationship? Int Urogynecol J Pelvic Floor Dysfunct. 2008;19:179-83.

18. Taithongchai A, Veiga SI, Sultan AH, Thakar R. The consequences of undiagnosed obstetric anal sphincter injuries (OASIS) following vaginal delivery. Int Urogynecology J. 2020;31:635-41. https://doi. org/10.1007/s00192-019-04033-5.

19. Jordan PA, Naidu M, Thakar R, Sultan AH. Effect of subsequent vaginal delivery on bowel symptoms and anorectal function in 
women who sustained a previous obstetric anal sphincter injury. Int Urogynecology J. 2018;29:1579-88.

20. Karmarkar R, Bhide A, Digesu A, Khullar V, Fernando R. Mode of delivery after obstetric anal sphincter injury. Eur J Obstet Gynecol Reprod Biol. 2015;194:7-10.

21. Cassis C, Giarenis I, Mukhopadhyay S, Morris E. Mode of delivery following an OASIS and caesarean section rates. Eur J Obstet Gynecol Reprod Biol. 2018;230:28-31.

22. Roos A-M, Thakar R, Sultan AH. Outcome of primary repair of obstetric anal sphincter injuries (OASIS): does the grade of tear matter? Ultrasound Obstet Gynecol. 2010;36:368-74.

23. Avery K, Donovan J, Peters TJ, Shaw C, Gotoh M, Abrams P. ICIQ: a brief and robust measure for evaluating the symptoms and impact of urinary incontinence. Neurourol Urodyn. 2004;23: $322-30$.

24. Laycock J (1994) Clinical evaluation of the pelvic floor. In: Pelvic Floor re-education, principles and practice. Springer (ed); pp 42-8.

25. Elharmeel SM, Chaudhary Y, Tan S, Scheermeyer E, Hanafy A, Driel ML van (2011) Surgical repair of spontaneous perineal tears that occur during childbirth versus no intervention. Cochrane Database Syst Rev. CD008534. https://doi.org/10.1002/14651858. CD008534.pub2.

26. Gommesen D, EAa N, Qvist N, Rasch V. Obstetric perineal ruptures - risk of anal incontinence among primiparous women
12 months postpartum: a prospective cohort study. Am J Obstet Gynecol. 2020;222:165.e1-165.e11. https://doi.org/10.1016/j. ajog.2019.08.026.

27. Evans E, Falivene C, Briffa K, Thompson J, Henry A. What is the total impact of an obstetric anal sphincter injury? An Australian retrospective study. Int Urogynecology J. 2020;31(3):557-66. https://doi.org/10.1007/s00192-019-04108-3.

28. Stock L, Basham E, Gossett DR, Lewicky-Gaupp C. Factors associated with wound complications in women with obstetric anal sphincter injuries (OASIS). Am J Obstet Gynecol. 2013;208: 327.e1-6.

29. Knight M, Chiocchia V, Partlett C, Rivero-Arias O, Hua X, Hinshaw K, et al. Prophylactic antibiotics in the prevention of infection after operative vaginal delivery (ANODE): a multicentre randomised controlled trial. Lancet. 2019;393:2395-403. https:// doi.org/10.1016/S0140-6736(19)30773-1.

30. Sioutis D, Thakar R, Sultan AH. Overdiagnosis and rising rate of obstetric anal sphincter injuries (OASIS): time for reappraisal. Ultrasound Obstet Gynecol. 2017;50:642-7.

Publisher's note Springer Nature remains neutral with regard to jurisdictional claims in published maps and institutional affiliations. 